\title{
Thermodynamic foundation of generalized variational principle
}

\author{
B.H. Sun \\ Institute of Mechanics and Technology and School of Civil Engineering \\ Xi'an University of Architecture and Technology, Xi'an 710055, China
}

\begin{abstract}
One open question remains regarding the theory of the generalized variational principle, that is, why the stress-strain relation still be derived from the generalized variational principle while the Lagrangian multiplier method is applied in vain? This study shows that the generalized variational principle can only be understood and implemented correctly within the framework of thermodynamics. As long as the functional has one of the combination $A\left(\epsilon_{i j}\right)-\sigma_{i j} \epsilon_{i j}$ or $B\left(\sigma_{i j}\right)-\sigma_{i j} \epsilon_{i j}$, its corresponding variational principle will produce the stressstrain relation without the need to introduce extra constraints by the Lagrangian multiplier method. It is proved herein that the $\mathrm{Hu}$-Washizu functional $\Pi_{H W}\left[u_{i}, \epsilon_{i j}, \sigma_{i j}\right]$ and $\mathrm{Hu}$-Washizu variational principle comprise a real threefield functional.
\end{abstract}

Keywords: variational principle, elasticity, Lagrangian multipliers, thermodynamics, entropy

\section{Introduction}

Variational principles have always played an important role in both theoretical and computational mechanics [1, 2, 8, 3, 4, 5, 6, 7, 9, 10, 12, 13, 14, $15,16,24,26,27,29,21,23,28,32,31,33,25,130,18,22,11,17,20,19$. Generalized variational mechanics began in the 1950s with the breakthrough works of Reissner [2] on two-field variational principles for elasticity problems, in which the displacement $u_{i}$ and stress $\sigma_{i j}$ are considered independent fields. The previous literature, however, considered only displacement $u_{i}$ as a single

\footnotetext{
Thermodynamic foundation of generalized variational principle Email addresses: sunbohu@xauat.edu.cn (B.H. Sun), +86 15001102877 (B.H. Sun) $U R L$ : imt.xauat.edu.cn (B.H. Sun)
} 
independent field. Reissner introduced a functional $F$ that is defined in terms of 12 arguments: six stresses $\sigma_{i j}$ and six strains $\epsilon_{i j}$ :

$$
F=\sigma_{i j} \epsilon_{i j}-B\left(\sigma_{i j}\right),
$$

where $B\left(\sigma_{i j}\right)$ is the elastic complementary energy density.

Reissner proved the following theorem: Among all states of stress and displacement that satisfy the boundary conditions of the prescribed surface displacement, the actually occurring state of stress and displacement is determined by the variational equation:

$$
\delta\left\{\iiint_{V} F d V-\iint_{S_{p}} \bar{p}_{i} u_{i} d S\right\}=0,
$$

where the symbol $V$ indicates the volume of the elastic body and $S_{p}$ indicates that the surface integrals are to be taken over that part of the surface only where the appropriate surface stress is prescribed.

In 1954, Hu published a paper [4] (its English version appeared in 1955 [6]) that borrowed the idea from Reissner [2] and successfully extended Reissner's two-field (displacement-stress) theory to a three-field (displacement-stressstrain) theory by introducing a functional $H_{U}$ given by

$$
H_{U}=A\left(\epsilon_{i j}\right)-f_{i} u_{i}-\sigma_{i j}\left(\epsilon_{i j}-\frac{1}{2} u_{i, j}-\frac{1}{2} u_{j, i}\right) .
$$

$\mathrm{Hu}[6])$ proved a theorem as follows:

$$
\delta\left\{\iiint_{V} H_{U} d V-\iint_{S_{\sigma}} \bar{p}_{i} u_{i} d S-\iint_{S_{u}} \sigma_{i j} n_{j}\left(u_{i}-\bar{u}_{i}\right) d S\right\}=0 .
$$

In 1955, Washizu [7] independently proposed the same functional and proved the same theorems as $\mathrm{Hu}[4,6]$.

Regarding the history of the generalized variational principle, Felippa [32] published a dedicated paper on the original publication of the generalized variational principle and showed that de Veubeke had developed a much more generalized variational principle in a report dated 1951 [3], in which four fields, namely, displacement, stress, strain, and surface force, were included. de Veubeke's four-field $\left(u_{i}, \sigma_{i j}, \epsilon_{i j}, t_{i}\right)$ theory can be presented as follows [32]: $\delta \Pi=0$, where the functional

$$
\begin{aligned}
\Pi_{V}\left[u_{i}, \sigma_{i j}, \epsilon_{i j}, t_{i}\right] & =\iiint_{V}\left[A\left(\epsilon_{i j}\right)+\sigma_{i j}\left(\frac{1}{2} u_{i, j}-\frac{1}{2} u_{j, i}-\epsilon_{i j}\right)-f_{i} u_{i}\right] d V \\
& -\iint_{S_{\sigma}} \bar{t}_{i} u_{i} d S-\iint_{S_{u}} t_{i}\left(u_{i}-\bar{u}_{i}\right) d S .
\end{aligned}
$$


The three-field standard form is obtained by setting $t_{i}=\sigma_{i j} n_{j}$ on $S_{u}$ a priori. Hence, Fellippa proposed that the canonical functional in Eq. 5 be called the de Veubeke-Hu-Washizu functional $\Pi_{V H W}$. This proposal has been confirmed by The History of the Theory of Structures Searching for Equilibrium [33].

In 1983, Chien [23], who was Hu's supervisor and communicated Hu's paper to both the Chinese Journal of Physics [4] and Science Sinica [6], pointed out that, regarding all publications and reports of Reissner [2], $\mathrm{Hu}[4,6]$ and Washizu [7] did not give any information on how to construct the functional. The formulation of the generalized variational seems mystical, and thus Chien indicated that the trial-and-error method was used when Reissner, $\mathrm{Hu}$, and Washizu formulated their functional [23, 28].

To derive the generalized functional in a systematic way, Chien proposed to formulate the functional by using the well-known method of Lagrangian multipliers [21]. This method can be described as follows [21, 23, 28]: Multiply undetermined Lagrange multipliers by various constraints and add these products to the original functional. Considering these undetermined Lagrange multipliers and the original variables in these new functionals as independent variables of variation, it can be seen that the stationary conditions of these functionals give these undetermined Lagrange multipliers in terms of original variables. The substitutions of these results for Lagrange multipliers into the above functional lead to the functional of these non-conditional variational principles.

With the help of the Lagrangian multipliers, in 1983 Chien [23] successfully reformulated the two-field functional, namely, $\Pi\left[u_{i}, \sigma_{i j}\right]$ and $\Pi\left[u_{i}, \epsilon_{i j}\right]$, which are called the Hellinger-Resissner functional [2] and De Veubeke functional [3], respectively. However, Chien [23] found that the constitutive relation between stress and strain cannot be included to form a three-field functional $\Pi\left[u_{i}, \epsilon_{i j}, \sigma_{i j}\right]$ due to the zero crisis of corresponding Lagrangian multipliers, as it is known to be impossible to incorporate this condition of constraint into a functional whenever the corresponding Lagrange multiplier turns out to be zero. Therefore, Chien claimed that the functional $H_{U}$ is not a three-field, but rather a two-field, functional. To address this point of view, Chien elegantly wrote a monograph on the generalized variational principle [28] and, to overcome the difficulty, he proposed a method of a higher-order Lagrange multiplier, a four-field functional $\Pi_{Q}$ that is suggested to be expressed as follows:

$$
\begin{aligned}
\Pi_{Q}\left[u_{i}, \sigma_{i j}, \epsilon_{i j}, \lambda\right] & =\iiint_{V} H_{U} d V-\iint_{S_{\sigma}} \bar{p}_{i} u_{i} d S-\iint_{S_{u}} \sigma_{i j} n_{j}\left(u_{i}-\bar{u}_{i}\right) d S \\
& +\iiint_{V} \lambda\left[A\left(\epsilon_{i j}\right)+B\left(\sigma_{i j}\right)-\sigma_{i j} \epsilon_{i j}\right] d V .
\end{aligned}
$$


Chien proved that for no zero $\lambda \neq 0$, the $\delta \Pi_{Q}\left[u_{i}, \sigma_{i j}, \epsilon_{i j}, \lambda\right]=0$ will produce balance equations, strain-displacement relations, stress-strain relations, and corresponding boundary conditions. Owing to the arbitrary nature of the Lagrangian multiplier $\lambda$, there are an infinite number of functionals $\Pi_{Q}\left[u_{i}, \sigma_{i j}, \epsilon_{i j}, \lambda\right]$.

Regarding Chien's questioning [23, 28], no explanation from $\mathrm{Hu}$, to the best of our knowledge, has been found in the literature. Because the formulation of the generalized variational principle has been recognized as a key contribution by a Chinese scholar to mechanics worldwide, and in particular, considering its importance in finite-element formulation, it is vital that Chien's question can be clearly answered. Otherwise, it will continue to cause confusion to both scholars and students. The task of answering this question has become a newcomer's responsibility.

\section{Chien's question on three-field variational principle}

To propose our understanding of the issue, a brief review of one-, two-, and three-field variational principles, as well as of Chien's question, is presented now.

Let $V$ be the volume of an elastic body, $S_{u}$ the boundary surface where displacement is given, and $S_{\sigma}$ the boundary surface where external force is given. Letting $S$ be the total boundary surface, then $S=S_{u}+S_{\sigma}$.

Assuming the body is subjected to the action of distributed body force $f_{i}(i=$ $1,2,3), S_{p}$ is the portion of the boundary surface subjected to the action of external surface force $\bar{p}_{i}$ and $S_{u}$ the portion of the boundary surface where the displacement $\bar{u}_{i}$ is given. Under statical equilibrium, the stress state in the body is denoted by stress tensor $\sigma_{i j}$. Displacement $u_{i}$, strain $\epsilon_{i j}$, and stress $\sigma_{i j}$ satisfy the following five conditions, that is,

$$
\sigma_{i j, j}+f_{i}=0, \quad(i n V)
$$

which is the balance equation, and $\sigma_{i j, j}=\frac{\partial \sigma_{i j}}{\partial x_{j}}$, where $j$ is a dummy index;

$$
\epsilon_{i j}=\frac{1}{2}\left(u_{i, j}+u_{j, i}\right), \quad(i n V),
$$

which is a strain-displacement relation;

$$
\begin{aligned}
\sigma_{i j}=\frac{\partial A\left(\epsilon_{i j}\right)}{\partial \epsilon_{i j}}, & (i n V), \\
\epsilon_{i j}=\frac{\partial B\left(\sigma_{i j}\right)}{\partial \sigma_{i j}}, & (\text { in } V),
\end{aligned}
$$


which are stress-strain relations;

$$
u_{i}=\bar{u}_{i}, \quad\left(\text { on } S_{u}\right)
$$

which is the boundary conditions for a given surface displacement; and

$$
\sigma_{i j} n_{j}=\bar{p}_{i}, \quad\left(\text { on } S_{\sigma}\right),
$$

which is the boundary conditions for a given external surface force; $n_{j}$ is normal unit vector of surface $S_{\sigma}$.

For a one-field potential functional,

$$
\begin{aligned}
\Pi\left[u_{i}\right] & =\iiint_{V}\left[A\left(u_{i, j}\right) d V-f_{i} u_{i}\right] d V \\
& -\iint_{S_{\sigma}} \bar{p}_{i} u_{i} d S-\iint_{S_{u}} \sigma_{i j} n_{j}\left(u_{i}-\bar{u}_{i}\right) d S .
\end{aligned}
$$

Its extreme condition $\delta \Pi\left[u_{i}\right]=0$ leads to the balance equation $\sigma_{i j, j}+f_{i}=0$ with constraints of $\epsilon_{i j}=\frac{1}{2}\left(u_{i, j}+u_{j, i}\right)$ and $\sigma_{i j}=\frac{\partial A\left(\epsilon_{i j}\right)}{\partial \epsilon_{i j}}$, boundary condition $u_{i}=\bar{u}_{i}$ on $S_{u}$, and $\sigma_{i j} n_{j}=\bar{p}_{i}$ on $S_{\sigma}$.

If one wishes to eliminate the constraint of strain-displacement relation $\epsilon_{i j}=\frac{1}{2}\left(u_{i, j}+u_{j, i}\right)$, according to Chien [23], a symmetric tensor of Lagrangian multiplier $\lambda_{i j}$ can be introduced and form a functional as

$$
\begin{aligned}
\Pi\left[u_{i}, \epsilon_{i j}\right] & =\iiint_{V}\left[A\left(\epsilon_{i j}\right)-f_{i} u_{i}\right] d V-\iint_{S_{\sigma}} \bar{p}_{i} u_{i} d S \\
& -\iint_{S_{u}} \sigma_{i j} n_{j}\left(u_{i}-\bar{u}_{i}\right) d S \\
& +\iiint_{V} \lambda_{i j}\left[\epsilon_{i j}-\frac{1}{2}\left(u_{i, j}+u_{j, i}\right)\right] d V .
\end{aligned}
$$

The Lagrangian multiplier $\lambda_{i j}$ can be determined by $\delta \Pi\left[u_{i}, \epsilon_{i j}, \lambda_{i j}\right]=0$, namely, $\lambda_{i j}=-\frac{\partial A\left(\epsilon_{i j}\right)}{\partial \epsilon_{i j}}$; therefore, the two-field de Veubeke functional is

$$
\begin{aligned}
\Pi\left[u, \epsilon_{i j}\right] & =\iiint_{V}\left[A\left(\epsilon_{i j}\right)-f_{i} u_{i}\right] d V-\iint_{S_{\sigma}} \bar{p}_{i} u_{i} d S \\
& -\iint_{S_{u}} \frac{\partial A\left(\epsilon_{i j}\right)}{\partial \epsilon_{i j}} n_{j}\left(u_{i}-\bar{u}_{i}\right) d S \\
& -\iiint_{V} \frac{\partial A\left(\epsilon_{i j}\right)}{\partial \epsilon_{i j}}\left[\epsilon_{i j}-\frac{1}{2}\left(u_{i, j}+u_{j, i}\right)\right] d V .
\end{aligned}
$$

If one wishes to carry on this process and eliminate the stress-strain relations, $\sigma_{i j}=\frac{\partial A\left(\epsilon_{i j}\right)}{\partial \epsilon_{i j}}$, one can do so by introducing another symmetric tensor of 
Lagrangian multiplier $\eta_{i j}$ to form a new functional as

$$
\begin{aligned}
\Pi\left[u_{i}, \epsilon_{i j}, \sigma_{i j}\right] & =\iiint_{V}\left[A\left(\epsilon_{i j}\right)-\iiint_{V} f_{i} u_{i}\right] d V-\iint_{S_{\sigma}} \bar{p}_{i} u_{i} d S \\
& -\iint_{S_{u}} \frac{\partial A\left(\epsilon_{i j}\right)}{\partial \epsilon_{i j}} n_{j}\left(u_{i}-\bar{u}_{i}\right) d S \\
& -\iiint_{V} \frac{\partial A\left(\epsilon_{i j}\right)}{\partial \epsilon_{i j}}\left[\epsilon_{i j}-\frac{1}{2}\left(u_{i, j}+u_{j, i}\right)\right] d V \\
& +\iiint_{V} \eta_{i j}\left(\sigma_{i j}-\frac{\partial A\left(\epsilon_{i j}\right)}{\partial \epsilon_{i j}}\right) d V .
\end{aligned}
$$

The Lagrangian multiplier $\eta_{i j}$ can be determined by $\delta \Pi\left[u_{i}, \epsilon_{i j}, \sigma_{i j}, \eta_{i j}\right]=0$, which leads to $\eta_{i j}=0$ and $\epsilon_{k l} \frac{\partial^{2} A\left(\epsilon_{i j}\right)}{\partial \epsilon_{i j} \partial \epsilon_{k l}}=0$, since $\frac{\partial^{2} A\left(\epsilon_{i j}\right)}{\partial \epsilon_{i j} \partial \epsilon_{k l}}>0$, thus giving an incorrect result $\epsilon_{i j}=0$.

If one examines the three-field functional $\Pi\left[u_{i}, \epsilon_{i j}, \sigma_{i j}, \eta_{i j}\right]$ carefully, one can find that there is only one term in the right containing stress tensor, namely, $\iiint_{V} \eta_{i j} \sigma_{i j} d V$, and there are no other terms to balance it in its variation, which means that $\delta \iiint_{V} \eta_{i j} \sigma_{i j} d V=\iiint_{V}\left(\delta \eta_{i j} \sigma_{i j}+\eta_{i j} \delta \sigma_{i j}\right) d V=0$ will definitely produce the result of $\eta_{i j}=0$. To overcome this, using the stress-strain relation, one can try to replace the term $\frac{\partial A\left(\epsilon_{i j}\right)}{\partial \epsilon_{i j}}$ with $\sigma_{i j}$, and hence the three-field functional becomes

$$
\begin{aligned}
\Pi\left[u_{i}, \epsilon_{i j}, \sigma_{i j}\right] & =\iiint_{V}\left[A\left(\epsilon_{i j}\right)-f_{i} u_{i}\right] d V-\iint_{S_{\sigma}} \bar{p}_{i} u_{i} d S \\
& -\iint_{S_{u}} \sigma_{i j} n_{j}\left(u_{i}-\bar{u}_{i}\right) d S \\
& -\iiint_{V} \sigma_{i j}\left[\epsilon_{i j}-\frac{1}{2}\left(u_{i, j}+u_{j, i}\right)\right] d V \\
& +\iiint_{V} \eta_{i j}\left(\sigma_{i j}-\frac{\partial A\left(\epsilon_{i j}\right)}{\partial \epsilon_{i j}}\right) d V .
\end{aligned}
$$

The Lagrangian multiplier $\eta_{i j}$ can be determined by $\delta \Pi\left[u_{i}, \epsilon_{i j}, \sigma_{i j}, \eta_{i j}\right]=0$, which leads to $\eta_{k l} \frac{\partial^{2} A\left(\epsilon_{i j}\right)}{\partial \epsilon_{i j} \partial \epsilon_{k l}}=0$, since $\frac{\partial^{2} A\left(\epsilon_{i j}\right)}{\partial \epsilon_{i j} \partial \epsilon_{k l}}>0$, again $\eta_{i j}=0$.

These results reveal that the stress-strain relation cannot be included in the functional, $\Pi\left[u_{i}, \epsilon_{i j}, \sigma_{i j}\right]$, by the Lagrangian multiplier method. In other words, it is impossible to remove all the constraints simply because the related Lagrange multiplier is equal to zero in the stationary condition. This Lagrangian multiplier method crisis was discovered by Chien in 1983 [23], when he published a monograph and provided a comprehensive discussion of the issue [28]. 


\section{Thermodynamic foundation of generalized variational principle}

For the sake of brainstorming on the stress-strain relation, a quick brief of constitutive theory from a thermodynamics perspective is presented here.

Combining the first law and second laws of thermodynamics [34, 35] leads to an equation defined in the current configuration as follows:

$$
\rho(\dot{\phi}+\eta \dot{\theta})-\boldsymbol{\sigma}: \dot{\boldsymbol{\epsilon}}+\frac{1}{\theta} \boldsymbol{q} \cdot \boldsymbol{\nabla} \theta \leq 0,
$$

where $\phi(\boldsymbol{\epsilon}, \theta)$ is the Helmholtz free energy, $\eta(\boldsymbol{\epsilon}, \theta)$ the entropy, $\theta$ the temperature, $\boldsymbol{\sigma}$ the stress tensor, $\dot{\boldsymbol{\epsilon}}$ the rate of deformation, and $\boldsymbol{q}$ the heat flux vector. Since $\dot{\phi}=\phi_{, \boldsymbol{\epsilon}}: \dot{\boldsymbol{\epsilon}}+\phi_{, \theta} \dot{\theta}=\phi_{, \boldsymbol{\epsilon}}: \dot{\boldsymbol{\epsilon}}+\phi_{, \theta} \dot{\theta}$, one can obtain

$$
\left(\rho \frac{\partial \phi}{\partial \boldsymbol{\epsilon}}-\boldsymbol{\sigma}\right): \dot{\boldsymbol{\epsilon}}+\rho\left(\frac{\partial \phi}{\partial \theta}+\eta\right) \dot{\theta}+\frac{1}{\theta} \boldsymbol{q} \cdot \boldsymbol{\nabla} \theta \leq 0,
$$

Note that the left-hand side of the inequality is a polynomial of degree 1 in $\dot{\epsilon}$ and $\dot{\theta}$. Because the inequality must hold for all choices of $\dot{\boldsymbol{\epsilon}}$ and $\dot{\theta}$, it will therefore be violated unless $\boldsymbol{\sigma}=\rho \frac{\partial \phi}{\partial \epsilon}$ and $\eta=-\frac{\partial \phi}{\partial \theta}$, which are the constitutive equations of simple materials $[34,35]$. The purpose of this brief review of the formulation of constitutive equations is to show that the thermodynamic relation,

$$
\rho(\dot{\phi}+\eta \dot{\theta})-\boldsymbol{\sigma}: \dot{\boldsymbol{\epsilon}}+\frac{1}{\theta} \boldsymbol{q} \cdot \nabla \theta \leq 0,
$$

has already contained the constitutive relations. In other words, the thermodynamic relation,

$$
\rho(\dot{\phi}+\eta \dot{\theta})-\boldsymbol{\sigma}: \dot{\boldsymbol{\epsilon}}+\frac{1}{\theta} \boldsymbol{q} \cdot \nabla \theta \leq 0,
$$

is an implicit form of constitutive equation.

For isothermal reversible thermodynamics, $\dot{\theta}=0$, one has

$$
\rho \dot{\phi}-\sigma: \dot{\epsilon}=0
$$

which can further be reduced to

$$
\rho d \phi-\sigma: d \boldsymbol{\epsilon}=\rho d \phi-\sigma_{i j} d \epsilon_{i j}=0 .
$$

If one replaces $\rho \phi$ by the potential energy density $A\left(\epsilon_{i j}\right)$, we have

$$
d A\left(\epsilon_{i j}\right)-\sigma_{i j} d \epsilon_{i j}=0,
$$

the equivalent form of which is the stress-strain relation:

$$
\sigma_{i j}=\frac{\partial A\left(\epsilon_{i j}\right)}{\partial \epsilon_{i j}}
$$


. Similar to the above-mentioned general case, $d A\left(\epsilon_{i j}\right)-\sigma_{i j} d \epsilon_{i j}=0$ implies the stress-strain relation.

Furthermore, since

$$
d A\left(\epsilon_{i j}\right)-\sigma_{i j} d \epsilon_{i j}=d A\left(\epsilon_{i j}\right)-\left[d\left(\sigma_{i j} \epsilon_{i j}\right)-\epsilon_{i j} d \sigma_{i j}\right]=0,
$$

and denoting

$$
d B=\epsilon_{i j} d \sigma_{i j}
$$

after integration one has

$$
A\left(\epsilon_{i j}\right)+B\left(\sigma_{i j}\right)-\sigma_{i j} \epsilon_{i j}=C .
$$

Applying conditions $A(0)=0$ and $B(0)=0$, thus $C=0$. Hence,

$$
A\left(\epsilon_{i j}\right)+B\left(\sigma_{i j}\right)-\sigma_{i j} \epsilon_{i j}=0,
$$

where $A\left(\epsilon_{i j}\right)$ and $B\left(\sigma_{i j}\right)$ are the potential and complementary energy density, respectively.

Following the above thinking, it is easy to know that a functional has included a stress-strain relation if it contains either the terms $\left[A\left(\epsilon_{i j}\right)-\sigma_{i j} \epsilon_{i j}\right]$ or $\left[B\left(\sigma_{i j}\right)-\sigma_{i j} \epsilon_{i j}\right]$.

In other words, if the structure of the functional was in the following form,

$$
\Pi\left[u_{i}, \epsilon_{i j}, \sigma_{i j}\right]=\iiint_{V}\left[\underline{A\left(\epsilon_{i j}\right)-\sigma_{i j} \epsilon_{i j}}+\ldots\right] d V+\text { Boundary tems }
$$

it implies that the stress-strain relation $\sigma_{i j}=\frac{\partial A\left(\epsilon_{i j}\right)}{\partial \epsilon_{i j}}$ is included. Because its variational $\delta \Pi\left[u_{i}, \epsilon_{i j}, \sigma_{i j}\right]=0$, namely, $\delta\left\{\iiint_{V}\left\{\underline{A\left(\epsilon_{i j}\right)-\sigma_{i j} \epsilon_{i j}}+\ldots\right\} d V+\right.$ Boundary tems $\}=$ 0 , leads to

$$
\left.\iiint_{V}\left\{\left[\frac{\partial A\left(\epsilon_{i j}\right)}{\partial \epsilon_{i j}}-\sigma_{i j}\right] \delta \epsilon_{i j}+\ldots\right\} d V+\delta \text { (Boundary tems }\right)=0
$$

and due to the arbitrary variation $\delta \epsilon_{i j}$, one therefore has the following stressstrain relation: $\frac{\partial A\left(\epsilon_{i j}\right)}{\partial \epsilon_{i j}}-\sigma_{i j}=0$.

Similarly, if the structure of the functional was in the following form,

$$
\Pi^{*}\left[u_{i}, \epsilon_{i j}, \sigma_{i j}\right]=\iiint_{V}\left[\underline{B\left(\sigma_{i j}\right)-\sigma_{i j} \epsilon_{i j}}+\ldots\right] d V+\text { Boundary tems. }
$$

it implies that the stress-strain relation $\epsilon_{i j}=\frac{\partial B\left(\sigma_{i j}\right)}{\partial \sigma_{i j}}$ is included. 
With this understanding, an examination of the following $\mathrm{Hu}$-Washizu functional is necessary:

$$
\begin{aligned}
\Pi_{H W}\left[u_{i}, \epsilon_{i j}, \sigma_{i j}\right] & \left.=\iiint_{V}\left\{\frac{A\left(\epsilon_{i j}\right)}{-} f_{i} u_{i}-\underline{\sigma_{i j}\left[\epsilon_{i j}\right.}-\frac{1}{2}\left(u_{i, j}+u_{j, i}\right)\right]\right\} d V \\
& -\iint_{S_{\sigma}} \bar{p}_{i} u_{i} d S-\iint_{S_{u}} \sigma_{i j} n_{j}\left(u_{i}-\bar{u}_{i}\right) d S
\end{aligned}
$$

The combination of the underlined terms in the above functional is exactly the term of $A\left(\epsilon_{i j}\right)-\sigma_{i j} \epsilon_{i j}$. Therefore, the Hu-Washizu functional $\Pi_{H W}\left[u_{i}, \epsilon_{i j}, \sigma_{i j}\right]$ has already included the stress-strain relation $\sigma_{i j}=\frac{\partial A\left(\epsilon_{i j}\right)}{\partial \epsilon_{i j}}$. The Hu-Washizu functional $\Pi_{H W}\left[u_{i}, \epsilon_{i j}, \sigma_{i j}\right]$ is a real three-field functional. This key point was not understood by $\mathrm{Hu}[4,6]$, Reissner [2], and de Veubeke [3] when they constructed their own generalized functional by the trial-and-error method. This situation is very similar to the formulation of the Schrödinger wave equation in quantum mechanics. The Schrödinger equation provides a way to calculate the wave function of a system and how it changes dynamically in time. However, the Schrödinger equation does not directly say exactly what the wave function is. For instance, Schrödinger originally viewed the electron's wave function as its charge density was smeared across space, but Born reinterpreted the absolute square value of the wave function as the electron's probability density distributed across space.

Of course, Chien's functional $\Pi_{Q}\left[u_{i}, \epsilon_{i j}, \sigma_{i j}\right]$ in Eq. 6 is not only a three-field functional, but also much more general one, since it contains all elements, such as $A(\epsilon i j), B\left(\sigma_{i j}\right)$, and $\sigma_{i j} \epsilon_{i j}$. The arbitrary nature of $\lambda$ provides some kind flexibility in constructing a generalized functional.

\section{Conclusions}

It has been shown in this study that the generalized variational principle can only be correctly understood and implemented within the framework of thermodynamics. As long as the functional has any one of the combination $A\left(\epsilon_{i j}\right)-\sigma_{i j} \epsilon_{i j}$ or $B\left(\sigma_{i j}\right)-\sigma_{i j} \epsilon_{i j}$, its corresponding variational principle can produce the stress-strain relation without the need to introduce extra constraints by the Lagrangian multiplier method.

It has been proved that the $\mathrm{Hu}$-Washizu functional $\Pi_{H W}\left[u_{i}, \epsilon_{i j}, \sigma_{i j}\right]$ is a real three-field functional and therefore that the $\mathrm{Hu}$-Washizu variational principle is a three-field variational principle. In addition, that Chien's functional $\Pi_{Q}\left[u_{i}, \epsilon_{i j}, \sigma_{i j}\right]$ is a much more general three-field functional has been confirmed. 
Owing to Chien's academic acumen, he discovered the problems and carried out meticulous research. His research inspired the author to think further on this issue. Although it was finally proved that the result of the $\mathrm{Hu}$-Washizu functional was only correct in form, the current understanding has risen to a new level, leading to the resolution of the historic academic controversy on the issue of constructing a three-field functional.

\section{Acknowledgments}

The author is honored to have benefited from personal connections with both Prof. Chien and Prof. Hu. Professor Chien supervised Prof. Kai-yuan Ye, the author's Ph.D. supervisor. Professor Hu was the committee chairman for the author's post-doctoral final progress report when he completed his postdoctoral research at Tsinghua University in 1991. In the great discovery of the generalized variational principle, both Prof. Qian and Prof. Hu made original contributions. Their academic thoughts are very important to our understanding of the generalized variational principle. Now that both Prof. Chien and Prof. $\mathrm{Hu}$ have passed away, if some valuable answer to the Qian question can be provided it can serve as the best tribute to both. Therefore, it is my privilege to dedicate this paper to the memories of Prof. Chien and Prof. Hu for their great contribution to the theory of the generalized variational principle. The author wish to express his gratitude to Prof. Felippa for his private communication and providing the copy of Fraeijs de Veubeke, B. M., Diffusion des inconnues hyperstatiques dans les voilures á longeron couplés, Bull. Serv. Technique de L'Aéronautique No. 24, Imprimerie Marcel Hayez, Bruxelles, 56pp (1951).

Availability of data: This study does not have any data.

Conflicts of interests: The author declares that there are no competing financial interests.

B.H. Sun: Conceptualization, methodology, formulations, formal analysis, funding acquisition, investigation, writing/original draft preparation, and writing/reviewing/editing all relevant works.

\section{References}

[1] Hellinger, E., Die allgemeinen Ansätze der Mechanik der Kontinua. Enzyklopädie der Mathematischen Wissenschaften, 4, Art. 30, pp. 654-655,(1914)

[2] Reissner, E., On a variational theorem in elasticity. J. Math. \& Phys. 29, 90-95, (1950). 
[3] Fraeijs de Veubeke, B. M., Diffusion des inconnues hyperstatiques dans les voilures á longeron couplés, Bull. Serv. Technique de L'Aéronautique No. 24, Imprimerie Marcel Hayez, Bruxelles, 56pp (1951)

[4] $\mathrm{Hu}, \mathrm{H} .-\mathrm{C}$. On the variational princinples in the theory of elasticity and the plasticity. Acta Physica Sinica, 10(3):259-290 (1954) (in Chinese) doi: 10.7498/aps.10.259.

[5] Naghdi, P.M., On a Variational Theorem in Elasticity and its Application to Shell Theory, J. Appl. Mech., Vol. 31, pp. 647-653(1954)

[6] $\mathrm{Hu}, \mathrm{H} .-\mathrm{C}$., On some variational methods on the theory of elasticity and the theory of plasticity, Scientia Sinica, 4, 33-54(1955)

[7] Washizu, K., On the variational principles of elasticity and plasticity, Aeroelastic and Structures Research Laboratory, Technical Report 25-18, MIT, Cambridge (1955)

[8] Reissner, E., On a variational theorem for finite elastic deformations. J. Math. \& Phys. 32, 129-153 ((1953)

[9] Gurtin, M.E., "Variational Principles for Linear Elastodynamics," Archive for Rational Mechanics and Analysis, Vol. 16, pp. 234-250, 1964.

[10] Reissner, E., A note on variational principles in elasticity. Int. J. Solids Struct. 1, 93-95(1965)

[11] Fraeijs deVeubeke, B. M., Displacement and equilibrium models, in Stress Analysis, ed. by O. C. Zienkiewicz and G. Hollister, Wiley, London, 145197(1965)

[12] Tonti, E., Variational Principles in Elastostatics, Mechanica, Vol. 2, pp. 201-208 (1967)

[13] Washizu, K., Variational Methods in Elasticity and Plasticity, Pergamon Press, New York(1968)

[14] Pian, T.H.H. and Tong, P., Basis of Finite Element Methods for Solid Continua, Int. J. of Num. Methods in Eng., Vol. 1:3-28, 1969.

[15] Fraeijs de Veubeke, B. A new variational principle for finite elastic deformations. Int. J. Eng. Sci. 10, 745-763 (1972) https://doi.org/10.1016/00207225(72)90079-1 
[16] Nemat-Nasser, S. , "General Variational Principles in Nonlinear and Linear Elasticity with Applications," Mechanics Today, Pergamon Press, Inc., Vol. 1,1973 .

[17] Fraeijs de Veubeke, B. M., Variational principles and the patch test, Int. J. Numer. Meth. Engrg., 8, 783-801(1974)

[18] Oden, J. T. and Reddy, J. N., On dual complementary variational principles in mathematical physics, Intern. J. Engrg. Sci., 12, 1-29 (1974)

[19] Ogden, R. W., A Note on Variational Theorems in Non-Linear Elastostatics, Proceedings of the Cambridge Philosophical Society, Vol. 77,609$615(1975)$

[20] Buffer, H., Generalized variational principles with relaxed continuity requirements for certain nonlinear problems, with an application to nonlinear elasticity. Comp. Meth. Appl. Mech. Eng. 19, 235-255(1979)

[21] Chien, W.Z., Variational Principles and Finite Element Method (Volume I), pp. 349-440, Science Press, Beijing (1980). (in Chinese).

[22] Oden, J. T. and Reddy, J. N., Variational Methods in Theoretical Mechanics, Springer-Verlag, Berlin (1982)

[23] Chien, W.Z., Method of higher-order Lagrange multiplier and generalized variational principles of elasticity with more general forms, Appl. Math. Mech. 4(2): 143-157(1983)

[24] Reissner, E., Variational principles in elasticity, manuscript of a chapter in: Handbook of finite element methods. London: McGraw-Hill(1983)

[25] Sun, B.H, Generalized variaonal principle in elasticity, B.Sc. Thesis, Xian Inst. of Highway, China (1983)(in Chinese).

[26] Reissner, E., Formulation of variational theorems in geometrically nonlinear elasticity. J. Eng. Mech. 110, 1377-1390(1984)

[27] Reissner, E. On mixed variational formulations in finite elasticity. Acta Mechanica 56, 117-125 (1985). https://doi.org/10.1007/BF01177113

[28] Chien, W.Z., Generalized Variational Princinples. Knowledge Publisher, Beijing (1985) (In Chinese). 
[29] Reissner, E. Some aspects of the variational principles problem in elasticity. Computational Mechanics 1, 3-9 (1986). https://doi.org/10.1007/BF00298634

[30] Sun, B.H., Generalized variational principle of electromagnatic continua, Congress Proc. of China Post-doctoral Fellows, 178-184, Peking University Press, 1991 (in Chinese).

[31] Felippa, C. A., A survey of parametrized variational principles and applications to computational mechanics, Comp. Meth. Appl. Mech. Engrg., 113, 109-139(1994)

[32] Felippa, C. A., On the original publication of the general canonical functional of linear elasticity, J. Appl.Mech. 67(1), 217-219, 2000.

[33] Karl-Eugen Kurrer, Philip Thrift, Ekkehard Ramm, The History of the Theory of Structures Searching for Equilibrium, Wilhelm Ernst \& Sohn.,Berlin, Germany (2018)

[34] Truesdell C., Toupin R. The Classical Field Theories. In: Flügge S. (eds) Principles of Classical Mechanics and Field Theory / Prinzipien der Klassischen Mechanik und Feldtheorie. Encyclopedia of Physics / Handbuch der Physik, vol 2/3/1. Springer, Berlin, Heidelberg(1960). https://doi.org/10.1007/978-3-642-45943-6_2

[35] Truesdell C., Noll W. The Non-Linear Field Theories of Mechanics. In: Antman S.S. (eds) The Non-Linear Field Theories of Mechanics. Springer, Berlin, Heidelberg (2004) https://doi.org/10.1007/978-3-662-10388-3_1 